\title{
Cybersecurity in Liberal Arts General Education Curriculum
}

\author{
Xenia Mountrouidou \\ Department of Computer Science \\ College of Charleston \\ Charleston, SC, USA \\ mountrouidoux@cofc.edu
}

\author{
Xiangyang Li \\ Information Security Institute \\ Johns Hopkins University \\ Baltimore, MD, USA \\ xyli@jhu.edu
}

\author{
Quinn Burke \\ Department of Education \\ College of Charleston \\ Charleston, SC, USA \\ burkeqq@cofc.edu
}

\begin{abstract}
Cybersecurity learning has been explored through different analytical lenses, across a range of grade levels and academic institutions. From attempts to standardize learning with accreditation to refining curricula and labs, there is currently considerable effort to create more programs in this discipline to address a million-job gap within the cybersecurity workforce. One primary challenge in cybersecurity education on the postsecondary level is offering experiential coursework to undergraduate students at liberal arts institutions. While such experiential coursework is already prevalent at research universities, smaller liberal arts institutions are still trying to gain a foothold into offering cybersecurity as a course of study. We address this issue with the design of an undergraduate course and cybersecurity learning modules that fit into the liberal arts education. First, we present the design of a general education course situated in a new educational paradigm, project CyberPaths, aiming at helping primarily undergraduate institutions with limited resources to introduce experiential cybersecurity learning. It takes advantage of experiential learning through a cloud infrastructure called Global Environment for Network Innovations (GENI). Then we describe our experience teaching this First Year Experience (FYE) course that exposed freshmen of different majors to cybersecurity. We present the design of pre- and post-course surveys, as well as focus group interviews that were used to evaluate students' learning experience. Student feedback and direct observation suggest that incorporating flexible cybersecurity modules into general education coursework can be an effective vehicle to demonstrate importance and key concepts of cybersecurity to a diverse student population.
\end{abstract}

\section{CCS CONCEPTS}

Social and professional topics $\rightarrow$ Computing Education Programs

\section{KEYWORDS}

Cybersecurity, General Education, Liberal Arts, GENI

ACM Reference format: Xenia Mountrouidou, Xiangyang Li, and Quinn Burke. 2018. Cybersecurity in Liberal Arts General Education Curriculum. In Proceedings of 23rd Annual ACM Conference on Innovation and

ermission to make digital or hard copies of all or part of this work for personal or claster fee provided that copies are not made or distributed for profit or commercial advantage and that copies bear this notice and the full citation on the first page. Copyrights for components of this work owned by others than the author(s) must be honored. Abstracting with credit is permitted. To copy otherwise, or republish, to post on servers or to redistribute to lists, requires prior specific permission and/or a fee. Request permissions from Permissionserocm redistribute to lists, requires prior specific
ITiCSE '18, July 2-4, 2018, Larnaca, Cyprus

$\odot 2018$ Copyright is held by the owner/author(s). Publication rights licensed to ACM.

ACM ISBN 978-1-4503-5707-4/18/07 \$15.00

https://doi.org/10.1145/3197091.3197110
Technology in Computer Science Education (ITiCSE'18). ACM, New York, NY, USA, 6 pages. https://doi.org/10.1145/3197091.3197110

\section{INTRODUCTION}

For the past few years, cybersecurity has received an increasing amount of attention from the media due to a variety of widely-publicized cyber-attacks, ransomware, ethical, and privacy concerns. At the same time, private enterprises and governments struggle to stay ahead of attackers as they face the reality that hiring and retaining talent in the information security field is a constant challenge [1]. Consequently, there is significant concern about a proposed million-job gap within the field [2], compounded by apprehension that the field lacks the means to adequately address such a steep gap.

On the other hand, colleges and their academic departments clearly have seen an opportunity for their graduates to gain prestigious, in demand job positions. Accordingly, their administrations are pushing to integrate cybersecurity into existing curricula for the creation of new majors, minors, and tracks in information security. Nationally, collaborative efforts between academia, government, and industry are increasing significantly, with one end goal to create new undergraduate, accredited programs in computer security [3], [4]. The ACM curriculum guide [5] has made cybersecurity a mandatory knowledge area, and Computer Science (CS) departments are increasingly developing various cybersecurity courses in their program of study. The National Security Agency (NSA) has put together a Center of Academic Excellence in Information Assurance program [6] that outlines knowledge unit areas.

To fill however the vast demand of cybersecurity talent, undergraduate programs ultimately need to explicitly focus on recruiting a greater diversity of students. Specially, primarily undergraduate (PUI) Liberal Arts (LIA) colleges are in a unique position to help to raise graduates who not only represent greater diversity in gender, race, and ethnicity, but also in their own educational background and interests.

The project CyberPaths [7] aims to take advantage of the general education arena to create a greater and more diverse 
population of cybersecurity professionals. The goal is not to turn every student who takes the initial general education class into a cybersecurity major; rather it is to offer students access to a baseline understanding of what cybersecurity is and why it matters, while simultaneously developing a new network for recruiting (and retaining) students with diverse qualifications and interests. Its vision is to develop not just technicians, but also policy makers and business professionals who understand cybersecurity fundamentals to protect themselves and their organizations. This paper reports our experience in one of these general education courses, a freshman experience course taught at the College of Charleston in spring and fall 2017.

\section{RELATED WORK}

There are several relevant education projects that aim to offer solutions to address the challenges of teaching cybersecurity. The Security Injections [8] project suggests teaching secure coding early, starting from the intro to programming CS1 and CS2 courses. SecKnitKit [9] is complementary to Security injections, adding cybersecurity modules to upper level CS courses, such as computer networks, operating systems, and databases. The project called SEED [10] is another example of using virtual machines (VMs) to teach cybersecurity concepts experientially. Unlike SecKnitKit, SEED does not aim at teaching cybersecurity concepts in every CS course, but only in specialized security courses. EduRange [11] is a project based on the Amazon cloud that aims to teach cybersecurity experientially. Weis et. al. [12] review a set of hands-on experiential learning cyber security exercises taught in liberal arts colleges and present their strengths and weaknesses.

Project CyberPaths distinguishes itself from these programs by focusing on bringing cybersecurity to students via nonintrusive general education coursework, while minimizing the requirement of investment in faculty and equipment. This project overcomes the lack of sanitized labs by adopting a cloud infrastructure, the Global Environment for Network Innovations (GENI) [13] to introduce experiential cybersecurity learning labs. These emulation labs on production computer networks offer real experimentation rather than VMs, in contrast to SecKnitKit and SEED. Furthermore, students do not have the burden of storing multiple images of VMs in their personal devices since they are using a cloud infrastructure. As an academic cloud, it offers flexibility unparalleled by commercial clouds such as EduRange's use of the Amazon cloud, which limits network attack and defense experimentation.

\subsection{General Education \& First Year Experience}

General education is a flagship of liberal arts colleges in order to develop students' perspectives and prepare well-rounded citizens with an appreciation of basic sciences, humanities and arts. One of CS departments' most promising forays into general education has been through their capacity to help students to solve problems more effectively through the formal and informal use of algorithms. Such reasoning has been termed

\footnotetext{
${ }^{1}$ http://blogs.cofc.edu/cyberpaths/modules/
}

"computational thinking" [14] and here we briefly review ongoing related efforts.

The Harvey Mudd College's CS1 courses introduced by Alvarado [15] aim to teach programming to all students regardless of their chosen major. In some cases, the main focus of CS in general education is on basic computer literacy, such as using the Microsoft Office Suite tools [16]. According to Perez et. al. [4] general education core in two-year community colleges may include a technology literacy course as well. Since CS used to be narrowly defined by the use of computer tools, the latest effort in colleges aims to introduce computational thinking courses in general education [14]. However, there is still resistance to accepting CS as part of the general education core since it may not strictly fit in one category, such as logic, scientific method, or empirical reasoning. Furthermore, computer science and cybersecurity are still considered by some to be specialized technical areas that may not be applicable to any other major.

The project CyberPaths attempts to change this perception and enforce that cybersecurity is an interdisciplinary field that can be embraced by a Liberal Arts core curriculum.

\section{GENERAL EDUCATION CYBER SECURITY COURSE DESIGN}

A First-Year Experience (FYE) course at the College of Charleston is a general education class that every freshman has to take. Any professor may teach an FYE course and there is flexibility on the topics to cover, as long as it satisfies the writing and research learning objectives of the general education curriculum. A professor who teaches this course may choose favorite topics related to his or her research area. In other cases, the course may revolve around broader interests, such as philosophy, arts, environmental, or civil issues.

We used the FYE general education course as a vehicle to experiment with the ideas of the project CyberPaths. Our objectives were:

i. Introduce non-CS majors to cybersecurity concepts that included both hard technology skills and interdisciplinary competencies,

ii. Facilitate undergraduate students to experience cyberattacks and technical security skills with realistic GENI labs, and

iii. Inspire a student of any major to contribute in the field of cybersecurity even if they did not choose to study CS.

The class was designed as a combination of traditional CS course assignments that include technical labs and a semester project, but in addition included literature readings, case studies, and writing essays. The contents of the class as well as the technical labs and assignments are available in public websites ${ }^{1,2}$. The format of the class was lectures combined with hands on exercises and technical labs, conversation on case studies, and peer instruction with Kahoot quizzes. We expand on three

\footnotetext{
${ }^{2}$ http://mountrouidoux.people.cofc.edu/FYE_CySec/index.html
} 
important components of the course: first, the semester project; second, the technical assignments, and third, the non-technical interdisciplinary case studies, as summarized in Figure 1.

One important feature of the class was a semester long project. The objective of the project was to teach students how to do research with online and library resources, write a technical document, and understand that regardless of their course of study they can contribute to the field of cybersecurity. The spring and fall semester courses included variations of this project. During spring 2017, we gave out an extensive list of topics revolving around security: Internet of Things (IoT), Mobile Technologies, Multimedia Technologies, etc. We then organized small chunks of scaffolding deliverables during the semester. Interestingly, the student projects focused on IoT security. Some students focused on the business aspects of cybersecurity, since the second most popular major among students was business. In the class taught the second time in fall 2017, the project was specific to the 2016 US election hacking, revolving around its technical and ethical aspects. The students researched and wrote a report on digital voting, media of communication, and elections in today's digital world.

One important goal of this course was to design technical assignments that were manageable and interesting to nontechnical students. We started with a series of CS unplugged ${ }^{1}$ activities on the basics of computing such as binary numbers and computer architecture. We then moved to command line, and Kali Linux tools for password cracking and traffic analysis. Kali is a Linux distribution that offers a collection of offensive and defensive security tools. Consequently, these hands-on, short, and manageable labs prepared students for their project CyberPathsnd GENI labs. We specifically emphasized command line skills and observed a steep learning curve, especially for non-CS majors.

The students completed several labs on GENI: (1). introduction to GENI, (2). analysis of a Denial of Service attack, (3). password cracking, and (4) ransomware. The third and fourth labs were only used in the fall 2017 course since their design was not yet finalized over spring 2017. For the first lab, students had to reserve two virtual machines and verify communication. For the second lab, we had the following goals: (i) Formulate a hypothesis and test it using traffic analysis techniques and Wireshark $^{2}$, (ii) Generate regular and attack network traffic and observe the differences between those, (iii) Experience a cyberattack and the overwhelming consequences to a small network. Interestingly, the third lab regarding password cracking, was exceptionally popular. It included a pivot machine where students would guess an easy password. Then students had to scavenge in files for encrypted and hashed passwords, decrypt those or use collisions to find the originals. Each cracked password led them to access the next machine. Finally, the ransomware lab was an example of social engineering, where the instructor "tricked" the students to use a Python script that encrypted their previously readable files. This lab initiated a conversation on malware and several ransomware cases in the healthcare sector that had recently appeared in news.

\footnotetext{
${ }^{1} \mathrm{http}: / /$ csunplugged.org/
}

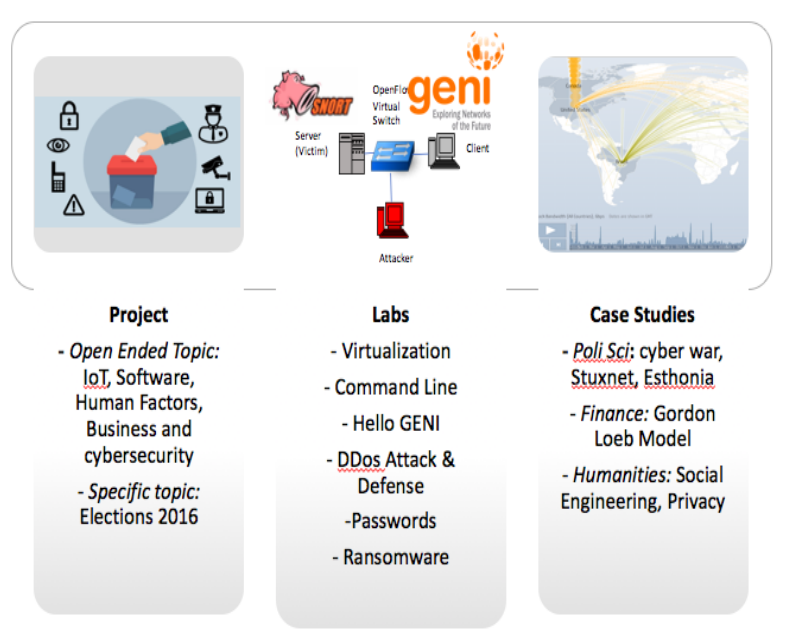

Figure 1: First Year Experience Course Design

For the case studies, we used modules on cyber conflict, ethics, and human factors in security from the project CyberPaths. These modules included a role play exercise where students apply political science, law, and disaster response principles in response to a real cyberattack, a research exercise on social engineering, and active discussions on ethics, offensive security, and privacy. These modules were combined with reading the literature book: "Future Crimes", by Mark Goodman, that inspired conversations and written assignments on privacy, IoT vulnerabilities, data anonymization compliance and laws, and other relevant topics.

\section{EVALUATION METHODS}

The project CyberPaths introduces cybersecurity in the general education liberal arts curriculum with the goal to increase the interest of students in the field and ultimately educate a larger number of diverse cyber security professionals. To this end, we aim to first understand what attracts these students to cybersecurity and what they themselves perceived to be the best methodologies to teach fundamental cybersecurity concepts. Thus, the following research questions guided our course surveys:

- In terms of recruitment, to what degree did the course attract both non-CS students and CS students through the first-year experience program?

- In terms of perception, what components of the course did students appreciate? Were there components they were less enthusiastic about? How did they perceive cybersecurity and computer science at course end?

- In terms of persistence, after taking the course, does student confidence in their own technical knowledge and cybersecurity increase? And, accordingly, do students plan to enter (or continue) with CS and specifically cybersecurity as a course of study?

\subsection{Survey Design}

All students were asked to complete pre- and post-surveys gauging their attitudes towards and confidence with digital

\footnotetext{
${ }^{2}$ https://www.wireshark.org/
} 
applications, computation, and, cybersecurity in particular. Adapted from Georgia Tech's computer science attitudes survey developed by Ni and Guzdial, the surveys gauged students' overall confidence and knowledge both pre- and post-course. The presurvey had a total of eight multiple choice questions related to social and educational background, 25 Likert scale questions about their familiarity and confidence using digital media and computational devices, and three open-ended questions related to cybersecurity and their goals for taking the course. The postsurvey had fewer questions, removing demographic questions but replicating the Likert-scale familiarity and confidence questions as well as the three open questions related to students' perspectives on the efficacy and content of the course.

The survey design revolved around the research questions from Subsection 4.1. To answer the first question, we needed to track students that switched their majors or ventured in the path of cyber security, which is part of our long-run evaluation. As an indicator of potential majoring in CS and security, we asked postsurvey questions regarding whether students planned to take additional CS courses and whether students made connections between their major and cyber security after the class. To understand which parts of the course students were most enthusiastic about, there was a set of open-ended questions about the hands-on assignments versus the case studies to measure students' preference in teaching techniques. To establish if the students' confidence increased after the class, we included a set of Likert questions in the pre- and post-surveys related to selfperception in problem solving and technical abilities. Naturally, performance in exams and technical projects is a strong indicator of gaining cyber security knowledge besides self-reporting in a survey, thus we included these in our study.

\subsection{Undergraduate Focus Groups}

In addition to pre- and post-surveys, a total of six undergraduates participated in a one-hour face-to-face focus group at the close of the semester. Structured around a total of seven questions gauging the progress in the introductory cybersecurity course, the focus group format was intended to be

more informal in nature and to encourage undergraduates to speak to each other about their experiences. The focus group discussions were recorded and subsequently transcribed and coded based on recurring themes in terms of course content, course clarity, and relevance [17]. As with the surveys, an external evaluator conducted the focus group and participants were informed that their names would be anonymized and their responses would in no way affect their grade in the course. Furthermore, Institutional Review Board approval was obtained for the surveys and focus groups.

\section{RESULTS}

In terms of enrollment, a total of 20 undergraduates enrolled in the FYE class in spring 2017 and 19 students the following fall. Twenty represents the maximum number of students for an FYE class at B. We present the merged survey results from both classes, of a total number of 39 participants. Among them, the most popular major was computer science, accounting for $62.5 \%$ in total. The second most popular major was business and economics (14\%), followed by humanities and other sciences, such as biology, psychology, English, and education majors. $44 \%$ of these students
Table I Profile of Entering Students

\begin{tabular}{|l|l|l|l|l|l|l|l|}
\hline $\begin{array}{l}\text { Programming } \\
\text { Experience? }\end{array}$ & \multicolumn{2}{l|}{$\begin{array}{l}\text { Programming } \\
\text { Semesters }\end{array}$} & \multicolumn{2}{l|}{ Math Years } & \multicolumn{2}{l|}{$\begin{array}{l}\text { Science } \\
\text { Years }\end{array}$} \\
\hline Yes & $42.2 \%$ & $\mathbf{0}$ & $57.7 \%$ & $\mathbf{3}$ & $3.3 \%$ & 3 & $10 \%$ \\
\hline No & $57.7 \%$ & $\mathbf{1}$ & $24.4 \%$ & $\mathbf{4}$ & $74.4 \%$ & 4 & $83.3 \%$ \\
\hline & & $>\mathbf{1}$ & $14.5 \%$ & $>\mathbf{5}$ & 22.2 & $>5$ & $6.6 \%$ \\
\hline
\end{tabular}

were female students, which was a rarity for a CS class. The population was primarily Caucasian, with $12.4 \%$ mixed race students, and $6 \%$ Hispanic students. The fall and spring classes had a different profile, the spring class was predominantly $(71 \%)$ female while the fall class included majority (83\%) of male, CS majors.

Table I shows the time that students had spent on programming, mathematics, and science in high school. It paints a picture of their strengths and weaknesses. While nearly $63 \%$ were declared CS majors, only $42.2 \%$ of the students had prior programming knowledge and out of these only $14.5 \%$ had more than one semester of programming education. As 19-year old Sara (pseudonym, as are all names hereon) in the focus group indicated of her own high school's computer science offerings, "There were some (CS courses). But I didn't take any....I didn't really know about it." This of course speaks to the reality that colleges are often picking up what high schools ought to be doing in terms of CS exposure, just as it suggests the need to introduce undergraduates early to computing coursework. However, despite the lower percentage of students who had prior CS exposure in high school, $74.4 \%$ had studied mathematics for four years and $83.3 \%$ had studied science for four years, which indicates that the population in the classes had a good base of fundamental science and mathematics knowledge.

In terms of the students' motivations for choosing the FYE course, it was encouraging that they did not only register for the course because this was a mandatory class, but they expressed strong interest in learning cybersecurity and CS. $87 \%$ of survey participants agreed or strongly agreed on the importance of cybersecurity as a course of study. The comments of the presurvey indicated that the students were looking forward to the class, they had positive attitude that is not always the case for taking a mandatory general education class, and that they took the class to learn how to protect themselves online.

\subsection{Perceptions: Student Responses to Individual Course Components}

In terms of gauging students' perceptions of the course, one significant product of the project CyberPaths is the GENI labs and general education modules that offer experiential learning to CS and non-CS majors alike. It was important for us to carefully assess students' experience with these materials and gather feedback on whether they could be improved. Thus, we aimed to answer this second research question: "Do students enjoy hands on more than case study assignments? And, if so, why do they prefer one over the other?"

Regarding the student experience running a realistic Denial of Service attack experiment and observing the results on the GENI infrastructure, $65 \%$ of the students mentioned in our postsurvey that it was positive. However, some student comments 
indicated that the technical parts of the lab were challenging. Specifically, students in the spring focus group interview mentioned that: "the GENI lab lasted multiple class periods" and "some of the instructions were confusing." This feedback helped us to understand that when using experiential learning labs that demand technical depth, students need intensive guidance.

During the fall offering of the class, we asked again the same question and observed a considerable improvement. This was due to revising the labs based on the student comments and suggestions. In fall 2017, we added the two aforementioned current labs on GENI, password cracking and ransomware. Specifically, the passwords lab was popular with several students mentioning in their comments that they enjoyed the lab "...because it was just trying to crack a password and find a solution to a problem." We plan to work on more "flag hunting" labs based on student comments that this was an exciting part of the class, as they mentioned: "(The lab was) building anticipation of the next big discovery in files to directories." Furthermore, it is encouraging that in the fall semester class $47 \%$ of the students said that the most exciting part of the whole class was the GENI labs. This may correlate to the CS majors being the majority of the class, thus we will keep measuring for improvements.

The results were encouraging when students self-assessed their cybersecurity knowledge and positive outcomes from the class, $87 \%$ of the students strongly agreed or agreed that after the class they understand what cybersecurity is and $83 \%$ strongly agreed or agreed that they understand the importance of the field.

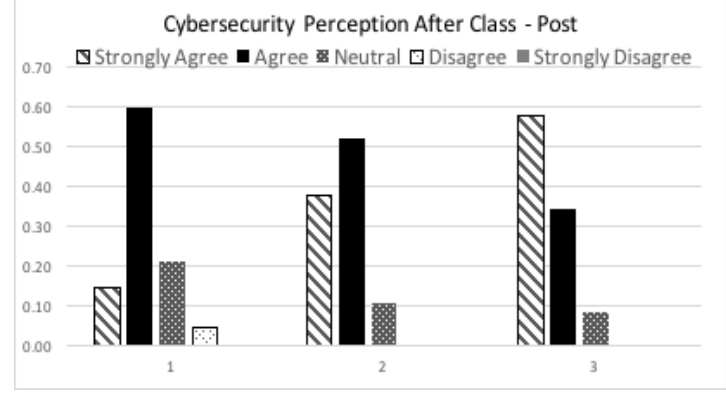

Figure 2: Gauging students' perceptions of cybersecurity knowledge (post-course survey; Spring \& Fall 2017 courses)

Figure 2 demonstrates the altogether positive response rates regarding students' perceptions of computer science and cybersecurity as relevant topics of study, as well as their growing competency in the disciplines. To Question1, "After this class, I have a better grasp of what computer science is all about," $75 \%$ of the 39 students strongly agreed or agreed with the statement. To Question 2, "After this class, I have a better grasp of what cyber security is all about," $89 \%$ strongly agreed or agreed with the statement. To Question 3, "After this class, I have a better understanding how cybersecurity plays a role in politics and society, in general," $92 \%$ strongly agreed or agreed with the statement.

Another positive result is that $78 \%$ of the students would recommend the class to another student. The course readings and activities, in fact, often led students to begin to feel themselves being exposed online as they begin to evaluate their own susceptibility to surveillance and potential identity theft. As 18year old Aaron explained, "The reading made me a bit paranoid!" Yet, despite such discomfort, students likewise appreciated the relevance of cyber-security to their own lives. "The information," explained 19-year old Lucia, "is definitely more applicable to life than other FYE courses, which are more like history and stuff like that...cyber security is part of our lives these days."

Lastly, an interesting difference was observed when students were asked to define cybersecurity in the pre- and post- survey. Only eight students gave a close to correct definition in the presurvey. This number doubled in the post survey with definitions entailing more precise and technical language. Overall, the class had a normal distribution of assignment and exam grades. The median final grade for the spring class was $85.6 \%$ and mean $89.1 \%$, the fall class final grade median was $91.3 \%$ and mean 88.3. Thus, both classes were able to demonstrate technical skills at a satisfactory level through technical assignments and tests. This, together with the success rate in the class answers the question: "After taking this class, does student knowledge in cyber security increase?".

\subsection{Persistence: Students' Self-Confidence \& Interest in Cybersecurity as a Future Course of Study}

In terms of students' perceptions of themselves as budding cybersecurity specialists and potential future computer scientists, our results were less fulfilling. Figure 3 (a) and (b) show the results of the pre- and post-course survey respectively, where students self-assessed their familiarity with technology and confidence in problem solving abilities. As evident with this top table, undergraduates did not enter the class with high confidence in their technology skills; only $42 \%$ considering themselves proficient with computers. On the other hand, they had a strong belief in their problem-solving skills at the outset of the course. In post survey results, this belief buoyed, with students indicating increased confidence in problem solving by $20 \%$ as evident with Question 2 (b) below. However, less encouraging is the fact that the rest of their confidence in technology skills compared to other students did not change significantly, as shown in Figure 3 (b) across Questions 1, 3, 4, and 5.

What was also disappointing was that despite students' increased sense of competency in cybersecurity (Figure 2), only $43 \%$ of the class indicated that they may take another CS class. To a degree, some of this reluctance may stem from the range of technical competency of entering students. As noted earlier, only $42 \%$ of undergraduates entered the FYE course with prior exposure to coding; a significant number of students over both terms had no prior exposure to programming basics and felt considerably less confident with the technical competencies. As one freshman Nora explained of her efforts in the final project assignment in spring 2017, in which the team was to hack a particular device or software program, "There was a big divide in my group because Fiona knew much more...Fiona had apparently been coding for three years, and already knows all this stuff about computers. I'm like in the middle, and then Bailey doesn't know anything. So poor Bailey didn't end up getting anything to do." 
To a degree, this range of varying computing competencies in student groups worked in that students undertook different roles in the creation of a final hack-able project (i.e., hacker, writer, presenter). However, going forward, we see the need to more explicitly identify the necessity of different roles within student groups and the fact that a student ought not feel less useful or left out due to lower coding competencies.

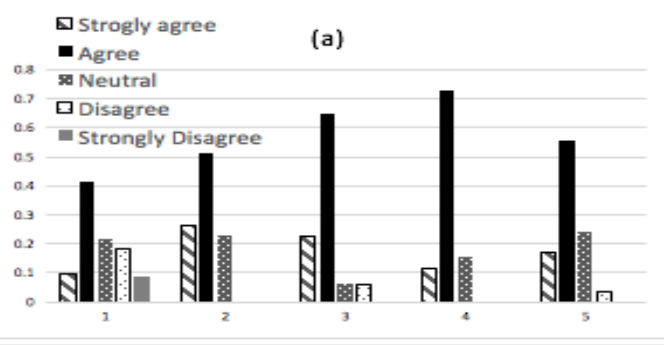

(b)

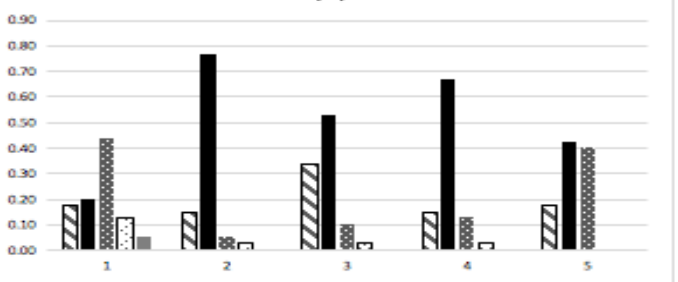

Q1 - Compared to other students in this class, I feel I know a lot about computers, Q2 - I am confident in my problem-solving ability, Q3 - I am confident in my ability to persist until a solution is found, Q4 - I am confident in meet unexpected challenges with success, Q5 - I enjoy being challenged by seemingly unsolvable situations or problems.

Figure 3: Gauging students' perceptions of their own (pre- and post-course survey; Spring \& Fall 2017 courses)

\section{CONCLUSIONS \& FUTURE WORK}

We have presented the results of teaching a first-year experience general education class on cybersecurity. In terms of our first research question around attracting a diverse group of undergraduates for the course, we largely succeeded. In terms of our second research question around student perceptions, our surveys show that students recognized the value of learning cybersecurity for their personal and organization benefits. One of our findings was that experiential technical learning excites students even though it may very well be challenging, because it rewards students with deeper understanding and sparks further interest among some to explore the field of cybersecurity. In terms of our third research question around student persistence, our results are less encouraging based on two iterations of the course. Interestingly, despite students' overwhelming recognition of the importance of cybersecurity and computer science, more generally, this recognition did not correlate with a perception that they themselves ought to continue to pursue it as an academic discipline.

In the future, we plan to make experiential learning more exciting and manageable with small chunking and Capture The Flag (CTF) type of assignments. We are currently working on a forensics lab and an IoT lab based on student feedback.
Furthermore, we will inject cybersecurity in more diverse general education classes such as finance, political science, and humanities, with goal to attract more non-CS majors to combine the field of cybersecurity with their discipline.

\section{ACKNOWLEDGMENTS}

This work was partially supported by the National Science Foundation Awards \#1700254, \#1525485.

\section{REFERENCES}

[1] (ISC) $)^{2}$, "2017 Global Information Security Workforce Study," [Online]. Available: http://bit.ly/2qVp6CF. [Accessed 2017].

[2] S. Morgan, "Zero-percent cybersecurity unemployment, 1 million jobs unfilled," CSO Online, 2016 September 2016. [Online]. Available: http://bit.ly/2cL50VM. [Accessed 2017].

[3] J.. Ekstrom, A. Parrish, E. Sobiesk, R. Raj, "ABET Supporter Session: Computing and CS Accreditation - What You Should Know," in 48TH ACM Technical Symposium on Computer Science Education (SIGCSE), March 8-11., Seattle, 2017.

[4] The 2011 ITiCSE Working Group on Information Assurance (IA) Education, "Information Assurance Education in Two- and Fouryear Institutions," in Proceedings of the 16th Annual Conference Reports on Innovation and Technology in Computer Science Education - Working Group Reports, Darmstadt, Germany, 2011.

[5] "ACM Joint Task Force on Cybersecurity Education," 2015. [Online]. Available: http://www.csec2017.org/. [Accessed 2016].

[6] "National Centers of Academic Excellence in Cyber Defense," 2016. [Online]. Available: http://bit.ly/2bJiCA2. [Accessed 2017].

[7] "Project CyberPaths," [Online]. Available: http://blogs.cofc.edu/cyberpaths/modules/. [Accessed 2017].

[8] "Security Injections @Towson - Cybersecurity Modules for Computer Science Courses," [Online]. Available: http://bit.ly/2G1llDq. [Accessed 2016].

[9] "Security Knitting Kit," [Online]. Available: http://bit.ly/2Dw4paM. [Accessed 2016].

[10] "SEED Labs," Syracuse University, [Online]. Available: http://bit.ly/2F1UKok.

[11] "EDURange: A Cybersecurity Competition Platform to Enhance Undergraduate Security Analysis Skills," [Online]. Available: http://bit.ly/2G2mk6c. [Accessed 2016].

[12] R. Weiss, J. Mache, and E. Nilsen, "Top 10 Hands-on Cybersecurity Exercises," Consortium for Computing Sciences in Colleges, vol. 29, no. 1, pp. 140--147, 2013.

[13] M. Berman, J. S. Chase, L. Landweber, A. Nakaod, M. Ott, D. Raychaudhuri, R. Ricci, and I. Seskar, "GENI: A federated testbed for innovative network experiments," Computer Networks, vol. 61, pp. 5-23, 2014.

[14] J. Wing, "Computational Thinking," Communications of the ACM, pp. 33-35, 2006.

[15] C. Alvarado, "CS1," [Online]. Available: http://bit.ly/2Dorg3T. [Accessed 2017].

[16] "CS 150, Personal Productivity Using Technology," [Online]. Available: http://bit.ly/2DzXcWb. [Accessed 2017].

[17] A. \&. J. C. Strauss, Basics of qualitative research: Techniques and procedures for developing grounded theory., Thousand Oaks, CA: Sage., 1998. 